\title{
Piste di ricerca sui rapporti tra l'università e le imprese a Bologna (1945-1968)
}

ISSN: 2282-4979

DOI: 10.12977/ere-

view134

Questo articolo è stato sottoposto a un processo di double blind peer review

I rapporti tra università e imprese nella Bologna del secondo dopoguerra e del successivo miracolo economico sono un interessante campo d'indagine. In questo contributo si vogliono mettere a fuoco alcune questioni di metodo e di merito, indicando anche le piste di ricerca relative alle relazioni intercorse fra il settore del packaging e la Facoltà di Ingegneria. Da queste connessioni sinergiche si giovarono sia il mondo accademico che quello imprenditoriale.

The relationships between university and business in Bologna during the post-war period and the subsequent economic development are an interesting research field. On this matter, the article focus on some methodological issues, also pointing to the research tracks related to the links between the packaging sector and the Faculty of Engineering. These synergistic connections favoured both the academic context and the entrepreneurial one.

La mente non è un vaso da riempire ma un legno da far ardere perché s'infuochi il gusto della ricerca e l'amore della verità.

Plutarco

\section{Premessa}

Negli ultimi decenni il dibattito politico è stato attraversato più volte da un tema complesso e tutt'altro che condiviso, e cioè il finanziamento privato alla ricerca pubblica, ovvero l'apporto economico che le imprese possono dare alle università 
in riferimento a specifici programmi di studio. Non è questa la sede per ripercorrere tale dibattito, né per enucleare le differenti posizioni in merito. Ci basti sapere che si tratta di un percorso storico di molto più lungo periodo, visto che i rapporti tra mondo economico e mondo accademico sono stati una costante. Senza voler risalire troppo addietro, limitandoci dunque all'età contemporanea, si può dire che nel XIX secolo le scuole di applicazione dell'Università di Bologna - fra le quali quella di Ingegneria, fondata nel 1877 - erano finanziate anche da consorzi e imprese del territorio, interessate ad avere tecnici con una formazione di qualità.

Nel presente contributo, vogliamo esaminare questo tema in via preliminare, in riferimento al periodo storico compreso fra il secondo dopoguerra e la fine degli anni Sessanta. Non è possibile presentare una ricerca compiuta o esaustiva, perché al momento l'indagine è in corso e necessita di ulteriori approfondimenti. Tuttavia, a partire da alcuni casi, svilupperemo delle prime considerazioni - di merito e di metodo - utili a perimetrare il tema e a collocarlo all'interno del dibattito storiografico. L'arco cronologico considerato è contraddistinto dal superamento delle difficoltà del dopoguerra - che in Emilia-Romagna furono particolarmente serie per via delle ingenti distruzioni subite - e dall'ingresso nel cosiddetto boom economico. Anche per queste ragioni, fra il 1945 e il 1968, il rapporto fra l'Università e le imprese si fece via via più stretto. Alcuni paragrafi iniziali illustreranno l'evoluzione dell'economia e della società bolognese nel periodo in questione, con numerosi riferimenti all'Ateneo, nell'intento di fornire al lettore alcune informazioni preliminari, indispensabili per cogliere le ragioni della ricerca in corso d'opera. Successivamente, in un più corposo paragrafo entreremo nel merito della questione, analizzando le piste di ricerca al momento più promettenti.

\section{L'economia bolognese}

Prima di approfondire il tema, è importante riepilogare brevemente la cornice storica entro la quale si inscrive la nostra analisi. Fiorente centro commerciale e produttivo durante il Medioevo, la città di Bologna visse un periodo di maggiore stagnazione economica in età moderna [Capitani (ed.) 2007], tanto che all'atto dell'Unità d'Italia il prodotto interno lordo della provincia era molto distante da quello del cosiddetto "triangolo industriale", e anzi era paragonabile a quello di varie realtà del Mezzogiorno [Felice 2007].

Le innovazioni in ambito rurale, concentrate fra Ottocento e Novecento, fecero da volano a una ripresa artigianale e industriale che nel primo dopoguerra $\mathrm{e}$ 
negli anni Venti avrebbe portato alla fondazione di numerose imprese destinate ad avere un importante futuro. La maggior parte di queste era attiva nel settore alimentare, come la Filicori-Zecchini e la Segafredo, che si aggiungevano ad altre già esistenti, quali la Majani, la Fabbri, la Buton [Battilani 2001]. Molte altre, invece, operavano nel settore della meccanica, che sarebbe diventato uno dei comparti industriali più importanti per la città. Ricordiamo la Calzoni, la Società Italo-svizzera, le Officine Maccaferri, le Officine Casaralta, la Minganti, la Gd, l'Acma, la Sasib, la Ducati, la Weber, solo per limitarci ad alcuni tra i nomi più importanti e noti. Da non trascurare, infine, il settore tessile, calzaturiero e chimico, ma anche le produzioni della Manifattura Tabacchi, della Hatù e della Arturo Gazzoni [Zamagni 1980].

Dopo la cesura della Seconda guerra mondiale - che per Bologna rappresentò la più grande tragedia in termini di morti e distruzioni - si ebbe una fase di ricostruzione morale e materiale molto rapida, che riattivò il tessuto economico produttivo nel giro di pochissimi anni. Da una parte gli aiuti americani del "piano Marshall" consentirono di ripristinare le principali infrastrutture, come strade, ponti, ferrovie, ecc., dall'altra gli industriali bolognesi attinsero a capitali propri e a finanziamenti di carattere pubblico, attraverso l'Imi, la Bnl e successivamente il Mediocredito regionale. In questa maniera, poterono ricostruire i capannoni bombardati, ricomprare i macchinari distrutti, pagare i primi stipendi, e quindi ricominciare a produrre per un mercato che nel frattempo si era lasciato alle spalle l'autarchia fascista e si stava ampliando a nuovi territori [Spagnolo 2001].

Erano i prodromi di quel boom economico che negli anni Cinquanta e Sessanta avrebbe completamente stravolto Bologna e la sua società, dando luogo all'eclissi dell'orizzonte rurale e a quell'affermazione della società industriale che in storiografia è chiamata «grande trasformazione» [Polanyi 1974]. Lo sviluppo, non certo limitato a Bologna, ma comune al resto d'Italia e a gran parte degli altri paesi europei, ebbe però un'intensità e un'ampiezza che in Emilia-Romagna furono superiori a quasi tutte le altre regioni. Vale a dire che quest'area, ancora fortemente agricola a metà del Novecento, si ritrovò ad essere un bacino manifatturiero di primo piano.

Basti pensare che in provincia di Bologna l'incidenza degli addetti all'agricoltura passò dal 38,0\% del 1951 al 7,7\% del 1981, con una diminuzione assoluta di più di 100.000 occupati; l'industria aumentava i posti di lavoro di circa 55.000 unità, mentre il settore dei servizi raddoppiava i propri addetti [Zamagni 1997]. Di fatto, quindi, l'agricoltura si modernizzava e si meccanizzava, e coniugava un aumento di produttività con un calo di addetti [Gobbo e Varni 1987]. Questi trovavano impiego sia nel sempre più dinamico settore industriale, che nel terziario, desti- 
nato a diventare un macrocomparto davvero consistente in termini di occupati e fatturato [Romei 2006].

Lo sviluppo industriale riguardò in primo luogo quei settori legati all'uso di tecnologie avanzate o alla produzione di beni di consumo durevoli. È il caso della meccanica, polo di eccellenza del bolognese, che in questi anni sviluppò sia il comparto "strumentale", legato alla produzione di macchine automatiche e tecnologie per altri settori industriali, che quello motoristico, in particolare nel segmento dei motocicli, sull'onda della motorizzazione di massa. Imprese storiche dell'area bolognese, come la Zanasi o la Cam nel packaging, la Ducati, la Moto Morini e la Malaguti, nella motoristica, la Weber nella produzione di componenti, consolidarono la propria posizione [Ferretti 2001; Menzani 2012]. Accanto a queste e ad altre importanti imprese capofila, gemmarono una serie di ditte artigianali, spesso fondate da tecnici, dipendenti e operai specializzati che avevano voluto "mettersi in proprio" o che erano stati licenziati "per rappresaglia", a seguito di attività politica o sindacale in fabbrica [Grappi e Mammana 1988; Arbizzani 2001].

Si crearono le premesse per un vero e proprio assetto distrettuale imperniato sul settore meccanico e metalmeccanico, in primo luogo rivolto alla produzione di macchine automatiche per il confezionamento. Maturò in questi anni l'espressione "packaging valley" a indicare che Bologna e il suo hinterland erano fortemente caratterizzati da imprese di questo settore. Contrariamente alla grande impresa manageriale americana, che alcuni decenni prima aveva costruito le proprie fortune sul fordismo, sulla catena di montaggio e su un'organizzazione razionale e accentrata, in questo caso prevalevano piccole e medie imprese legate da rapporti di subfornitura, di contoterzismo, di collaborazione formale e informale, che in maniera straordinariamente efficace erano però in grado di contare sempre più sui mercati internazionali [Menzani 2011; Pedrocco 2013].

\section{L'Università e le dinamiche demografiche felsinee}

L'Università di Bologna è considerata la più antica del mondo occidentale, e la sua fondazione è convenzionalmente datata nel 1088 , per opera di un certo Irnerio. Attualmente vanta diverse sedi decentrate, 11 scuole, 33 dipartimenti, e soprattutto 87.000 studenti. Ma nell'immediato dopoguerra questa dimensione di massa era ancora molto distante, e l'Ateneo di Bologna aveva numeri assolutamente più contenuti. Da un lato l'istruzione universitaria era un fenomeno ancora abbastanza elitario - basti pensare che nel 1950 l'Istat censiva 231.000 studenti universitari in tutta Italia, contro 1.700 .000 attuali -, e dall'altro il campo 
disciplinare era meno articolato e complesso di quello attuale. Ma soprattutto, l'università insisteva su un contesto demografico ancora acerbo e non raccoglieva iscrizioni da fuori regione in misura così elevata come sarebbe stato successivamente.

Nel 1936, la città di Bologna aveva circa 280.000 abitanti, ma il trend mostrava una crescita, a seguito dei processi di urbanizzazione che avevano caratterizzato il suo territorio negli anni fra le due guerre, delle politiche a favore della famiglia messe in atto dal regime fascista, ma anche dei progressi in campo igienicosanitario, che avevano concorso a ridurre la mortalità infantile e ad allungare la vita media delle persone [Dal Panta e Scalone 2006]. Anche nell'ambito di questa tendenza, la seconda guerra mondiale rappresentò una cesura, perché causò numerosissime vittime e indusse molti cittadini a sfollare in campagna. Non solo, ma l'aleatorietà degli eventi e la separazione fisica tra donne e uomini, perché molti di questi erano al fronte, portò ad un vero e proprio crollo delle nascite nella prima metà degli anni Quaranta.

La fine del conflitto, il graduale ritorno alla normalità e il rinnovato clima di fiducia nel futuro indussero molte giovani coppie a sposarsi e ad avere dei bambini, cosicché tra il 1945 e il 1950 Bologna fu caratterizzata da un forte incremento della natalità, a compensare anche la decrescita del quinquennio precedente. Nel 1951, i residenti erano saliti a 340.000 , principalmente a seguito di quello che fu chiamato "baby boom" [Micheli 1999]. Si tratta di un dato importante nella nostra narrazione. Infatti, il divario in termini di natalità fra prima e seconda metà degli anni Quaranta diventava molto più netto. E così, a Bologna e provincia il numero di nuovi nati tra il 1945 e il 1950 fu in proporzione molto superiore alla media nazionale [Finzi e Tassinari 1980]. Nei primi anni Sessanta, quindi, Bologna era una delle città italiane in cui la percentuale di adolescenti e preadolescenti compresi fra i 10 e i 15 anni era superiore a quella di quasi tutte le altre città italiane. Basti pensare che questa generazione avrebbe avuto fra i 18 e i 23 anni nel 1968, e non a caso - non certo solo per questa ragione - Bologna fu una delle realtà urbane più coinvolte nelle lotte studentesche.

In aggiunta a tutto ciò, la crescita della popolazione significò un allargamento della domanda di beni di consumo, di abitazioni, di strutture sociali come scuole e ospedali, e quindi comportò un ampliamento delle periferie [Parisini 2010]. Nei primi anni Sessanta, Bologna aveva iniziato un processo di graduale erosione delle aree agricole, e già si potevano intravvedere alcuni punti di contatto con l'hinterland, per cui dal centro città si poteva raggiungere Casalecchio di Reno o San Lazzaro di Savena o Castel Maggiore senza quasi attraversare aree di campagna [Gavioli 2003]. La città cresceva e con essa i servizi, la sua economia, e 
l'importanza nel panorama italiano. La conseguenza più vistosa di tutto ciò fu il generale e rapido miglioramento del tenore di vita della popolazione, in particolare delle classi lavoratrici. L'aumento del reddito procapite, che a Bologna all'incirca triplicò fra il 1950 e il 1970, si tradusse in una fortissima espansione dei consumi privati, tanto che in riferimento a quel periodo si è usata l'espressione di "società del benessere", o anche, con una sottile polemica, di "civiltà dei consumi" [Crainz 1996].

All'epoca, il capoluogo emiliano era una fucina di iniziative e progetti, culturali, urbanistici e imprenditoriali: nel 1963 erano stati inaugurati l'aeroporto e l'ospedale Maggiore, erano stati dismessi i tram a favore dei più moderni autobus e filobus, era iniziata la costruzione dell'Ospedale Malpighi e di Palazzo Affari, il cui cantiere, l'anno seguente sarebbe stato affiancato da quello del complesso fieristico [Ferri 2011]. In particolare crebbero vertiginosamente le percentuali di reddito destinate all'abbigliamento, agli articoli per la casa, e al tempo libero. Fra questi, vanno anche annoverati una serie di prodotti rivolti ai giovani, che mai prima di allora erano stati considerati così insistentemente come consumatori [Castronovo 2010].

In un contesto di questo genere, anche l'università si attrezzava per accogliere un crescente numero di studenti e per fare i conti con un tessuto economico molto più vivace, nel quale l'industria sarebbe stata il macrosettore di riferimento. L'analfabetismo era soltanto un lontano ricordo e le nuove generazioni erano sempre più scolarizzate e affamate di letture, per cui anche il mercato editoriale si adeguava a questo genere di domanda.

A Bologna si leggeva mediamente molto di più che altrove. Nel 1964 era inaugurata nei locali di Palazzo Strazzaroli, in piazza Porta Ravegnana, la libreria Feltrinelli, che a lungo sarebbe stata uno dei principali punti d'incontro dei lettori di Bologna. Nell'aprile dello stesso anno, presso il Palazzo del Podestà, si tenne la prima edizione della Fiera del libro per l'infanzia e la gioventù, come si chiamava inizialmente la Fiera del libro per ragazzi, ancora oggi esistente e giunta alla cinquantaquattresima edizione [Menzani 2013]. E varie istituzioni e culture politiche cittadine si mostravano molto sensibili verso il tema dell'istruzione giovanile e della lettura. Nel 1958, ad esempio, su impulso del Consiglio provinciale, fu istituito il Consorzio per il servizio della lettura e del prestito librario, che avrebbe iniziato la sua attività nel 1959. Scopo dell'ente era diffondere la lettura in tutti i centri della provincia nei quali il Comune non poteva impiantare o mantenere una biblioteca propria, o in quelli in cui si voleva integrare la biblioteca esistente, rappresentando di fatto il primo esperimento italiano di diffusione libraria ad ampio raggio [Bongiovanni, Franzoni e Vignali 2010]. 
Tutte queste dinamiche economiche e sociali rivelano che la società civile attorno all'università maturava bisogni formativi nuovi, più specialistici, aggiornati $\mathrm{e}$ con volumi maggiori rispetto al passato perché il numero degli studenti sarebbe cresciuto. Non solo la curva demografica felsinea avrebbe portato un'ondata di nuovi iscritti, ma Bologna era diventata anche un centro molto appetibile per residenti fuori regione. La crescita della domanda e dell'offerta didattica si accompagnava a una parallela implementazione della ricerca scientifica, sostenuta da nuovi bisogni euristici e da una volontà di investire in innovazione che si legava idealmente ai paralleli desiderata del mondo imprenditoriale.

\section{L'Università, l'amministrazione pubblica e le istituzioni intermedie}

Qualsiasi attività imprenditoriale, progetto culturale o iniziativa di carattere pubblico che parte da un territorio specifico deve presto o tardi relazionarsi con gli enti locali e con le istituzioni intermedie, o per lo meno con alcune di esse [Barbareschi 2006]. L'importanza di questo rapporto dipende nello specifico dalla natura della proposta in questione, ma in generale si può sostenere che il ruolo della pubblica amministrazione non può mai considerarsi marginale, e anzi in numerosi casi appare decisivo [Dogliani e Gaspari (eds.) 2003]. Fatta questa premessa, bisogna dire che l'amministrazione comunale bolognese del secondo dopoguerra e degli anni del miracolo economico è stata considerata da vari studiosi italiani ed esteri, di ieri e di oggi, come un modello degno di un certo interesse. Questo ha fatto sì che si alimentasse la rappresentazione - in parte fondata, in parte mitizzata - di una cultura amministrativa felsinea tra le migliori a livello europeo, sotto il profilo dell'utilizzo delle risorse, della progettualità, della lungimiranza, delle capacità di cogliere le opportunità e di governare una travolgente trasformazione [Baldissara 2004].

Il cosiddetto "modello emiliano", che ha avuto in Bologna uno dei casi più eclatanti e noti, è stato a lungo un riferimento e un esempio di "buona amministrazione", e di sinergie tra sviluppo industriale, costruzione del welfare, partecipazione popolare, progresso civile e sociale [Brusco 1982; Anderlini 1986]. Una delle principali leve di questo approccio amministrativo stava nel fornire servizi al cittadino in misura tale da rappresentare una forma di integrazione salariale. Si trattava di asili nido, di trasporto pubblico urbano, di biblioteche, di infrastrutture, di programmi assistenziali in ambito socio-sanitario, tutti erogati a prezzi ben al sotto di quelli di mercato o in forma addirittura gratuita. In questa maniera, questa integrazione salariale consentiva di evitare eccessivi e laceranti contrasti 
sul piano della rivendicazione sindacale, per lo meno nei confronti della piccola e media impresa, e di armonizzare, quindi, investimenti imprenditoriali, sviluppo economico e aumento dei consumi [De Maria (ed.) 2014]. Il costo del welfare locale era poi ripagato da una crescita complessiva delle attività produttive che andava ad alimentare il gettito fiscale [Alaimo 2002].

Queste ricadute positive sulla qualità della vita si ripercossero anche sullo sviluppo dell'ateneo, che del resto beneficiò anche del fervore delle altre istituzioni intermedie, ossia di quegli organismi locali di natura privata - come le associazioni di categoria, le banche, i sindacati o le organizzazioni religiose - e di natura pubblica, come le imprese municipalizzate, oppure di composizione mista, come le camere di commercio o i consorzi di bonifica. Si tratta di un insieme particolarmente vasto ed eterogeneo che riunisce una serie di soggetti che, a vario titolo, paiono importanti per lo sviluppo sociale ed economico di un territorio. Da una parte, forniscono servizi alla cittadinanza o a una parte di essa, dall'altra catalizzano beni relazionali e valori etici come la fiducia, l'onestà, la solidarietà [Arrighetti e Seravalli 1999].

\section{Una prima pista di ricerca: il settore del packaging}

Il settore del packaging - ampiamente descritto nel secondo paragrafo - è probabilmente uno di quelli che può fungere da case study in una ricerca sui rapporti fra mondo accademico e mondo economico. Naturalmente non è l'unico. Anche il settore agroalimentare o quello motoristico possono offrire in tal senso più di uno spunto. Tuttavia, il contesto delle macchine automatiche è già stato molto studiato, e sono emersi in tal senso vari riferimenti all'università.

Oggi, le grandi realtà bolognesi dell'automazione industriale - e cioè i gruppi Ima, Marchesini, Coesia/Gd, Sacmi - rappresentano un'eccellenza tecnologica a livello internazionale, con soluzioni assolutamente all'avanguardia. Come tali, hanno rapporti con la ex Facoltà di Ingegneria' dell'Università di Bologna e con vari altri atenei italiani, come l'Università di Modena e Reggio Emilia o il Politecnico di Milano, solo per citarne alcuni, ma anche con un crescente numero di università estere, dislocate in quei contesti nei quali questi gruppi manifatturieri possiedono le loro filiali produttive.

Tuttavia, la storia della cosiddetta packaging valley non è stata fin dalle origini interessata da un rapporto con la Facoltà di Ingegneria. Anzi, fino al secondo 
dopoguerra era assolutamente prevalente il legame con gli istituti tecnici e professionali, a iniziare da quello con la scuola Aldini-Valeriani [Campigotto, Curti, Grandi e Guenzi (eds.) 2000]. Gli ingegneri diventarono importanti protagonisti nel settore delle macchine automatiche a partire dagli anni Sessanta, quando il livello di complessità delle tecnologie aumentò e non era più sufficiente l'estro dei diplomati o lo "sbuzzo" degli autodidatti della prima ora [Curti e Grandi 1997]. Anzi, alcune testimonianze ci dicono che il rapporto fra l'Ateneo di Bologna e le imprese di questo settore è stato agli esordi un poco burrascoso o comunque episodico. Solo negli ultimi decenni è maturata una più stretta collaborazione fra l'università e le principali imprese del packaging, con programmi specifici, convenzioni per stage o tesi di laurea in azienda, master co-progettati e simili. In questa maniera, la precedente e ricorrente critica di un lacunoso rapporto tra la Facoltà di Ingegneria e le imprese nelle quali avrebbero lavorato gli ingegneri è venuta sostanzialmente meno [Menzani 2015]. Un'interessante pista da percorrere è proprio la ricostruzione storica di queste relazioni, che furono cruciali per la maturazione del comparto, ma anche per orientare la ricerca ingegneristica verso ambiti saldamenti ancorati alle necessità delle aziende del territorio. Bisognerebbe recuperare fonti in grado di dirci quali furono i primi contratti formali, che esiti diedero, come furono gestiti i brevetti, quale fu l'impegno economico da un lato e dall'altro, e anche quali furono i tempi di progressivo rinsaldamento di queste relazioni. Ad oggi conosciamo ancora poco di tutto ciò, e per lo più in termini qualitativi, cioè senza un numero sufficiente di dati relativi all'ammontare dei finanziamenti e ai costi della ricerca. Tuttavia, sappiamo che uno dei primi rapporti importanti fra packaging valley e Facoltà di Ingegneria dell'Università di Bologna è da imputare alla Gd.

La «Fabbrica italiana motociclette G.D.» - come si chiamava in origine - nacque nel 1923, per iniziativa dell'avvocato Mario Ghirardi, che finanziava interamente questo progetto imprenditoriale, e dell'ing. Guido Dall'Oglio, che apportava il bagaglio brevettuale. Inizialmente la società, che prendeva il nome dalle iniziali dei due fondatori, era una piccola officina che produceva artigianalmente motocicli, alcuni dei quali - come accadeva frequentemente all'epoca - erano impiegati in competizioni sportive. Fu un successo immediato, che giunse grazie alle numerose vittorie e ai tanti piazzamenti dei piloti in sella alle moto $\mathrm{Gd}$, tanto che la marca divenne ben presto molto nota nella cerchia degli appassionati [Campigotto, Grandi e Ruffini 2008]. A seguito di dissapori tra Ghirardi e Dall'Oglio, quest'ultimo usciva dalla ditta e la Gd cessava gradualmente la produzione seriale, per occuparsi solamente di riparazioni e di fabbricazione conto terzi o su commessa. Nel 1934, la società fu salvata dal fallimento da una piccola cordata 
guidata dal noto antifascista bolognese Leonildo Tarozzi, che ripianò i debiti e fece nuovi investimenti per riattivare la produzione motoristica.

Gli effetti furono inferiori alle aspettative, e nel 1939 la società fu venduta a Enzo Seràgnoli, imprenditore bolognese attivo nel settore del commercio delle granaglie e dei trasporti, propenso a indirizzare la Gd verso la produzione di autocarri. Invece lo scoppio della guerra sconvolse completamente questi progetti, e lo stesso Seràgnoli si persuase che fosse più opportuno spostare il core business verso un altro genere di produzione, dato che la motoristica richiedeva investimenti sempre più ingenti. Infatti, nell'immediato dopoguerra, il mito della $125 \mathrm{Gd}$ era tornato a splendere per qualche mese, in occasione di alcune motociclistiche, in particolare, alla "gimkana" di Reggio Emilia e nella prova del chilometro lanciato. Contemporaneamente, però, l'ingresso nel settore di alcuni colossi della meccanica, come la Piaggio, ma anche la Breda, la Macchi e la Caproni, nonché il ritorno in auge di alcuni marchi storici quali Guzzi, Gilera e Bianchi, avevano suggerito di non insistere con la motoristica [Petroni 1998, pp. 69-85].

Enzo Seràgnoli era perplesso di fronte alla scelta di abbandonare un comparto promettente come quello della costruzione dei veicoli, giacché era certo che la motorizzazione avrebbe avuto un ruolo di assoluto rilievo nello sviluppo economico dell'Italia democratica, ma volle dare fiducia all'intraprendenza del cugino Ariosto Seràgnoli, un tecnico particolarmente eclettico che nel frattempo era stato assunto in azienda, che invece insisteva nel giudicare il packaging come un settore estremamente promettente. In particolare, si pensò di avviare una produzione di macchine automatiche per l'incartamento di tavolette di cioccolata e di altri analoghi prodotti dolciari. Ariosto Seràgnoli, oltre alle indubbie qualità tecniche, aveva anche buone capacità organizzative, che rivelò nella scelta dei primi consulenti, i quali iniziarono a riunirsi nel nuovo ufficio aperto in via della Zecca. Qui si incontrarono con una certa frequenza alcuni fra i più grandi esperti di meccanica della Bologna del secondo dopoguerra, come il prof. Gino Morandi, docente di Macchine all'Università di Bologna, o l'ing. Renato Taino, preside dell'Istituto Alberghetti di Imola. La maggior parte dei brevetti depositati fra il 1946 e il 1950 derivò da queste collaborazioni, in lunghe riunioni, anche serali, durante le quali si confrontavano le competenze teoriche di Morandi e Taino con il genio innovatore di Ariosto Seràgnoli, e alle quali assistevano pochi altri stretti collaboratori, come Riccardo Mattei, destinato a una lunga carriera in Gd in qualità di direttore tecnico². 
In questo contesto, la Gd riuscì progressivamente a modellare la nuova identità produttiva e nel 1946, quando la Fiera di Milano riaprì i locali, tra gli oltre 2.500 espositori vi era anche un suo stand, con una macchina confezionatrice, la 2002, «incartatrice automatica idraulica regolabile per tavolette di cioccolato» ${ }^{3}$. Era un prototipo molto elementare, prodotto lavorando alacremente - giorno e notte sui disegni del prof. Morandi e sulle intuizioni di Ariosto Seràgnoli. Dal successo di quella macchina sarebbe dipeso l'intero futuro della $\mathrm{Gd}$, che aveva la necessità di riprendersi da un periodo abbastanza negativo. La 2002 fu molto apprezzata, non tanto perché si basasse su meccanismi particolarmente geniali o innovativi, quanto perché concreta ed efficace, in grado di svolgere il proprio compito con diligenza e zelo. Fu una vera e propria boccata d'ossigeno per l'azienda, che fece capire come il settore del packaging potesse regalare soddisfazioni, e che consentì il prosieguo della progettazione.

E allora si fece la prima Fiera Campionaria di Milano - racconta Alfonso Tomba -; quella diede il la, diede l'input alla macchina automatica. Ce ne chiesero tante ed ebbe prevalenza la scelta della macchina automatica, secondo una tesi di Ariosto Seragnoli che già allora diceva: «A fare delle motociclette sono capaci tutti». [...] Allora [Enzo] Seragnoli [...] fece una scelta. E fu una scelta non priva di rammarico $[\ldots]$ e spesse volte c'erano dei rimpianti nell'ambito di questo boom motoristico.

Si deve tenere presente che a poca distanza dall'officina Gd vi era lo stabilimento della Ducati, un'azienda di motociclette, che negli stessi anni ebbe un considerevole sviluppo. Ma Ariosto Seragnoli era convinto delle proprie valutazioni, godeva della fiducia del cugino Enzo, e immediatamente intraprese la realizzazione di altre macchine che sfruttavano i principi della 2002, e che sono generalmente note come la "serie 2000", nome che richiamava il concetto di modernità tecnologica e l'impronta d'avanguardia. In questa "serie 2000", si distinsero la 2000/B, per l'avvolgimento dei saponi, la 2004/B per i biscotti, la 2006 e la 2007, rispettivamente dosatore e mescolatore per sostanze in polvere, la impacchettatrice 2008, e la 2010 per l'incarto di cioccolatini e pralines. Tutte furono realizzate anche con la consulenza tecnica del prof. Morandi.

In questa fase di esordio nel comparto del packaging, la forza della Gd derivò dal fatto di aver compreso che il limite di molte macchine in commercio stava nella 
eccessiva specializzazione, per cui erano in grado di effettuare un solo tipo di incarto, con misure standard e limitate. Dunque, ci si propose l'obiettivo tecnico di realizzare e di offrire alla clientela delle incartatrici capaci di effettuare più tipi di confezionamento, con la sostituzione semplice e veloce di alcuni particolari, per avvolgere prodotti di misure e forme diverse. In pratica, si volevano creare poche macchine per soddisfare molte esigenze ${ }^{6}$. Nel 1947, dunque, la Gd rendeva noto alla Camera di commercio di Bologna di aver iniziato a costruire anche «macchine automatiche idrauliche incartatrici per prodotti dell'industria alimentare, chimica, ecc. [e] macchine dosatrici e mescolatrici»; e pochi anni dopo, la Camera di commercio certificava che la Gd risultava «abituale costruttrice di macchine sviluppatrici, incartatrici, cellofanatrici» .

L'incontro con il prof. Morandi avrebbe poi portato a un rapporto più strutturato con la Facoltà di Ingegneria, che avrebbe accompagnato tutto il successivo sviluppo dell'azienda. Infatti, dopo alcune difficoltà iniziali, la scelta del packaging si rivelò particolarmente azzeccata per la Gd e in poco tempo arrivarono ordini da tutta Italia e poi anche dall'estero. Gli anni del cosiddetto miracolo economico fecero da sfondo a uno sviluppo aziendale di assoluto rilievo, che proseguì anche negli anni Sessanta, quando - su commessa dei Monopoli di stato - si realizzarono le prime macchine per il confezionamento delle sigarette, fra le quali una cellofanatrice particolarmente rapida ed efficiente, denominata 4350/pack. Ma il vero successo nel cosiddetto tobacco packaging venne nei primi anni Settanta, con la realizzazione dell'impacchettatrice $\mathrm{x}-1$, che a parità di tempo confezionava circa il doppio di pacchetti rispetto alle macchine della concorrenza [Capecchi 1990].

L'idea rivoluzionaria che sottendeva al successo della $\mathrm{x}-1$ era una chiusura del pacchetto attorno al mandrino che non limitava l'estrazione delle sigarette dal medesimo; le bionde erano condotte verso il cartoncino «trasversalmente», in maniera che andassero a impattare sul «lato lungo» anziché «di punta» ${ }^{8}$. In questa maniera, l'urto era ripartito su una superficie più ampia, non provocava danni al prodotto, e consentiva velocità maggiori. Si trattava di una modifica apparentemente banale, ma di fatto vincente, che fece arrivare ordini da tutti i continenti, da parte di multinazionali del tabacco come la Philip Morris, la Reynold's, la Bri- 
tish and American Tobacco, solo per citare alcuni tra i principali clienti [Serafini 2014].

Rapidamente, la Gd divenne un punto di riferimento mondiale nel settore del confezionamento delle sigarette, con oltre duemila addetti, e sedi e filiali in molti paesi esteri, dagli Stati Uniti al Brasile, dall'Inghilterra a Hong Kong. Questa proiezione sui mercati internazionali si accompagnava a un adeguamento della struttura societaria, con l'ingresso di nuovi manager, attenti non solamente all'imprescindibile campo dell'innovazione tecnologica - nella quale la Gd continuava a mostrarsi all'avanguardia - ma anche all'ambito commerciale, a quello finanziario e alle sfide della responsabilità sociale. E naturalmente il rapporto con la Facoltà di Ingegneria divenne ancora più stretto.

\section{Una seconda pista di ricerca: la Menarini}

Un'altra impresa che ebbe rapporti con la Facoltà di Ingegneria dell'Università di Bologna nel corso degli anni sessanta è la Menarini, poi Bredamenarinibus, e di recente confluita in Industria italiana autobus. La Menarini e C. fu fondata a Bologna nel 1919 e, nonostante il nome suggerisse una proprietà collettiva, il titolare era unicamente Ettore Menarini, carrozziere e meccanico. L'impresa aveva sede in una stalla riadattata, fuori porta San Felice, in via del Chiù. All'inizio si riparavano solamente carrozzerie, poi con l'acquisto di alcuni macchinari si avviò anche la produzione, su commesse della Fiat. Si trattava delle carrozzerie (o di parti di esse) delle nuove vetture che la casa torinese aveva lanciato con successo sul mercato: la 501, la 503 e la 520. Ma per buona parte degli anni Venti, l'attività principale provenne ancora dalla realizzazione di "carrozze da turismo", trainate da cavalli. Era un lavoro svolto in collaborazione costante con il cliente (che seguiva da vicino i lavori), e di stampo decisamente artigianale: basti pensare che per costruire una carrozza occorrevano dai quattro ai sei mesi. Dal 1925, in una nuova sede in viale Berti Pichat n. 10, iniziò la produzione di carrozzerie per trasporti collettivi e usi industriali - autocarri, autobus, furgoni, cabine di trazione - sempre in collegamento con la Fiat. Era un mercato in rapida espansione e all'epoca più importante di quello strettamente automobilistico. Per quasi tutti gli anni Trenta, infatti, la produzione nazionale di vetture per trasporti collettivi fu superiore a quella di autoveicoli [Boschi 1970].

Agli inizi del conflitto mondiale, la Menarini era insediata su una vasta area di circa $3.500 \mathrm{mq}$, di cui circa 2.500 coperti. Vi lavoravano approssimativamente centocinquanta addetti, quasi tutti in sede, anche se vi erano alcuni distaccamenti, in particolare una succursale in via Balbo n. 33 e una rimessa in via S. Donato 
n. $160^{9}$. I locali, dotati di magazzini sotterranei, vennero ulteriormente ampliati nel 1942, quando si costruì nelle adiacenze un nuovo deposito di circa 800 mq. Durante gli anni della guerra si registrò una preoccupante fuoriuscita della manodopera specializzata, attratta dalle più alte paghe di altre imprese, che non erano vincolate dai prezzi bloccati delle commesse belliche. Invece alla Menarini, il $40 \%$ della produzione avveniva su ordinativi pubblici. Questa percentuale aumentò nelle fasi finali del conflitto, allorché la ditta produsse anche carrozzerie per autoambulanze, carri-officina e mezzi militari, per conto del Ministero dell'Aeronautica, dell'Officina militare delle trasmissioni di Roma, della Croce rossa italiana ${ }^{10}$.

Tra il 1944 e 1945, la fabbrica venne centrata ripetutamente dai bombardamenti e a pochi mesi dalla Liberazione dovette interrompere la produzione. Questa non poté ricominciare nemmeno subito dopo il 25 aprile, perché per un breve periodo lo stabilimento venne requisito dagli Alleati. Solamente nell'autunno del 1945, Ettore Menarini ebbe la possibilità di riprendere appieno il proprio ruolo imprenditoriale, affiancato più incisivamente dai figli. Dal matrimonio con Argia Atti, infatti, Ettore aveva avuto cinque maschi: Arnaldo (1906-2003), Dante (1909-1983), Giorgio (1911-1999), e i due gemelli Carlo (1923-1999) ed Eugenio (1923-2014).

Il primogenito Arnaldo, ingegnere, iniziò a lavorare autonomamente nel settore edile e non ebbe mai a che fare con l'impresa paterna. Anche Carlo, laureatosi in medicina, intraprese un percorso professionale diverso da quello del genitore. Gli altri tre figli, invece, - Dante, Giorgio ed Eugenio - furono coloro che portarono avanti la ditta. Dal punto di vista legale, nel 1941 la società aveva assunto il nome di Carrozzeria Menarini Bologna s.n.c., ed erano stati fatti soci Dante e Giorgio, mentre Eugenio entrò nella società solo nel 1951, anno in cui il padre Ettore ne fuoriuscì completamente [Biscaretti di Ruffia 1952]. Dante e Giorgio si erano diplomati alle Aldini-Valeriani; Eugenio, invece, terminati gli studi di ragioneria, si era iscritto alla Facoltà di Economia e commercio, anche se gli impegni di lavoro gli impedirono di conseguire la laurea. Il primo a entrare nell'azienda paterna era stato Dante che non ancora diciottenne aveva iniziato a frequentare i locali dell'officina. In seconda battuta era stato accolto Giorgio, forte anche di alcune brevi esperienze in Belgio e Francia, alle dipendenze di aziende meccaniche. Per ultimo entrò Eugenio, proprio all'indomani della Liberazione. 
La ripresa postbellica fu tutto sommato rapida. Sul finire del 1946 la Menarini riusciva a produrre già un autobus al mese, e nel 1952 arrivava addirittura a sei o sette. La svolta che fece decollare l'azienda arrivò proprio in quegli anni. Fu un'intuizione di Dante Menarini, che comprese che le carrozzerie degli autobus, all'epoca in legno, potevano essere realizzate in metallo a prezzi ugualmente vantaggiosi. Grazie ad alcuni contatti con imprese svizzere all'avanguardia nella produzione di profilati leggeri, Dante progettò un primo modello di carrozzeria in metallo. Fu un successo, che però poté essere visto solo parzialmente dal padre Ettore, che moriva a Bologna il 4 luglio 1952. Di lì in avanti l'azienda sarebbe stata condotta solamente dai tre figli: il direttore generale sarebbe stato Dante, il direttore tecnico Giorgio e il direttore amministrativo Eugenio".

Nelle seconda metà degli anni Cinquanta l'officina venne spostata verso la periferia, in località San Sisto, in uno stabilimento più ampio che sarebbe stato ulteriormente rinnovato di lì a qualche anno. Nel 1961 la ditta aveva oltre trecentosettanta operai, e una quarantina tra impiegati, intermedi e dirigenti. Al Salone dell'auto di quell'anno presentò per la prima volta la carrozzeria Essediemme, destinata ad avere un largo successo negli anni Sessanta e Settanta. L'Essediemme si caratterizzava per una minore tara, per le porte elettrocomandate, per un maggior isolamento acustico, e per l'intercambiabilità di molti pezzi in casi di guasto. In questa fase storica si irrobustirono i rapporti commerciali e collaborativi con le principali case automobilitistiche nazionali, in primis la Fiat, ma anche la Lancia e l'Alfa Romeo. Contemporaneamente si iniziarono a produrre anche i rimorchi. È in questa fase che - stando a quanto dichiarato da Eugenio Menarini-1'azienda prese contatto con la Facoltà di Ingegneria. Era interessata soprattutto ad approfondire la ricerca sui materiali, che in un certo senso rappresentavano il principale tratto innovativo della produzione. In secondo luogo, venne preso in considerazione anche il tema dell'automazione:

Anche se il nostro lavoro era soprattutto artigianale, la questione dell'innovazione ci interessava molto. Io avevo fatto l'università e sapevo che proprio all'università c'erano delle competenze che potevano rivelarsi utili per un salto di qualità. Così prendemmo contatto con alcuni docenti e ci facemmo consigliare sui materiali da impiegare e poi sui sistemi di apertura automatica delle porte. Gli autobus erano un prodotto che affascinava anche loro e collaboravano volentieri. 
Negli anni Settanta, la Menarini diventò famosa per essere una delle imprese italiane dove più forte era lo scontro tra la proprietà e le forze sindacali. Per diversi anni, la produzione procedette a singhiozzo, per via dei numerosi scioperi. Per far fronte alle rivendicazioni sindacali, Eugenio Menarini, che dal 1975 era alla guida dell'Assoindustriali di Bologna e vicepresidente nazionale della Federmeccanica, optò per un disimpegno su questi fronti e per un ritorno in azienda dove il clima diventava ogni giorno sempre più teso. Il 31 gennaio 1978 le Formazioni comuniste combattenti in collaborazione con Prima linea compirono un attentato alla casa di Dante Menarini. Nello stesso anno, il direttore della Menarini veniva gambizzato da alcuni terroristi dell'estrema sinistra. Ma fu l'ultimo atto intimidatorio di una qualche rilevanza. Sulla fine degli anni Settanta, il graduale svelenimento dello scontro politico-sindacale e il successivo rifluire dell'ondata brigatistica contribuirono alla normalizzazione all'interno della Menarini. Questa, nel frattempo, era diventata una società per azioni, e, nel 1979, aveva acquisito e inglobato le Officine Meccaniche Automobili di Granarolo (Omag) ${ }^{13}$.

Ma un lutto turbò questa riacquisita serenità aziendale: Dante Menarini moriva il 13 aprile 1983. La presidenza passò inizialmente al figlio Sandro e poi, dopo pochi mesi, a Giorgio Menarini. Alla morte di Dante, la ditta occupava settecentocinquanta dipendenti e produceva circa sessanta carrozzerie di autobus al mese. Da pochissimo aveva iniziato la fabbricazione di interi autobus, in cooperazione con alcune altre ditte meccaniche. È probabile che anche quest'ultimo passo fosse stato compiuto a seguito di un qualche progetto tecnico realizzato o discusso con uno o più docenti della Facoltà di ingegneria, ma occorrerebbe verificare nell'archivio dell'azienda.

I casi della Gd e della Menarini sono probabilmente emblematici rispetto a dinamiche di più ampio respiro. Nella Bologna del secondo dopoguerra e del successivo boom economico in molte occasioni si posero le basi per nuove e più durature collaborazioni tra mondo economico e mondo accademico. Si tratta di aspetti che meritano di essere indagati perché spesso si trattò di relazioni sinergiche, che diedero un valore aggiunto sia alla ricerca universitaria - che ottenne più risorse e che poté declinarsi su ambiti applicativi nuovi - sia alle aziende felsinee, che si giovarono di apporti di grande qualità, che in molti casi furono alla base dell'acquisita capacità di competere in maniera vincente sul piano internazionale. 


\section{Bibliografia}

Alaimo A. 2002, Un'altra industria? Distretti e sistemi locali nell'Italia contemporanea, Milano, Franco Angeli

Anderlini F. 1986, Modello padano: localismo e modernizzazione, Bologna: Il Mulino

Arbizzani L. 2001, La Costituzione negata nelle fabbriche: industria e repressione antioperaia nel Bolognese, 1947-1966, Bologna: Pass

Arrighetti A. e Seravalli G. (ed.) 1999, Istituzioni intermedie e sviluppo locale, Roma: Donzelli

Baldissara L. 2004, I comunisti bolognesi e il buongoverno municipale: programmi, propaganda e rappresentazioni nella costruzione di un modello, in De Bernardi A., Preti A. e Tarozzi F. (eds.) 2004, Il Pci in Emilia-Romagna: propaganda, sociabilità, identità dalla ricostruzione al miracolo economico, Bologna: Clueb

Barabaschi B. 2006, Qualità della pubblica amministrazione e sviluppo delle società locali, Milano: Franco Angeli

Battilani P. 2001, Il percorso impossibile. Dal laboratorio artigiano alla grande impresa nel settore alimentare: Bologna, in Amatori F. e Colli A. (eds.) 2001, Comunità di imprese. Sistemi locali in Italia tra Ottocento e Novecento, Bologna: Il Mulino

Biscaretti di Ruffia C. 1952, Carrozzieri di ieri e di oggi, Torino: Anfia

Bongiovanni M.L., Franzoni G. e Vignali G. (eds.) 2009, Il consorzio provinciale di pubblica lettura di Bologna, Bologna: Provincia e Assessorato cultura e pari opportunità

Boschi S. 1970, Carrozzeria Menarini. Cinquantenario. 1920/1970, Bologna: Righi

Brusco S. 1982, The Emilian Model: Productive Decentralisation and Social Integration, "Cambridge Journal of Economics", 2

Campigotto A., Curti R., Grandi M. e Guenzi A. (eds.) 2000, Prodotto a Bologna. Una identità industriale con cinque secoli di storia, Bologna: Renografica

Campigotto A., Grandi M. e Ruffini E. 2008, Moto bolognesi degli anni ’20, Vimodrone: Nada

Capecchi V. 1990, L’industrializzazione a Bologna nel Novecento. Dal secondo dopoguerra ad oggi, in Tega W. (ed.) 1990, Storia illustrata di Bologna, vol. V, Bologna contemporanea: gli anni della democrazia, Milano: Aiep

Capitani O. (ed.) 2007, Bologna nel Medioevo, Bologna: Bononia University Press

Castronovo V. 2010, L'Italia del miracolo economico, Roma-Bari: Laterza

Crainz G. 1994, Padania. Il mondo dei braccianti dall'Ottocento alla fuga dalle campagne, Roma: Donzelli

Crainz G. 1996, Storia del miracolo italiano. Culture, identità, trasformazioni fra anni Cinquanta e Sessanta, Roma: Donzelli

Curti R. e Grandi M. (ed.) 1997, Per niente fragile. Bologna capitale del packaging, Bologna: Compositori 
De Maria C. (ed.) 2014, Il modello emiliano nella storia d'Italia. Tra culture politiche e pratiche di governo locale, Bologna: Bradypus

Del Panta L. e Scalone F. 2006, Sviluppo demografico, urbanizzazione e flussi migratori in Provincia di Bologna tra XIX e XX secolo, "Metronomie: ricerche e studi sul sistema urbano bolognese", 32-33

Dogliani P. e Gaspari O. (eds.) 2003, L'Europa dei comuni. Origini e sviluppo del movimento comunale europeo dalla fine dell'Ottocento al secondo dopoguerra, Roma: Donzelli

Felice E. 2007, Divari regionali e intervento pubblico: per una rilettura dello sviluppo in Italia, Bologna: Il Mulino

Ferretti R. 2001, Reti di imprese e sistema economico locale. Industria meccanica e comparto motoristico a Bologna (1919-1971), in Amatori F. e Colli A. (eds.) 2001, Comunità di imprese. Sistemi locali in Italia tra Ottocento e Novecento, Bologna: Il Mulino

Ferri A. (ed.) 2011, Bologna 1900-2000: cronache di un secolo, Bologna: Bononia University Press

Finzi R. e Tassinari F. 1980, La società, in Zangheri R. (ed.) 1980, Bologna, Roma-Bari: Laterza

Gavioli M. 2003, Lungo la via Emilia: stagioni pianificatorie e governo delle trasformazioni a Bologna, Modena e Reggio Emilia, in Parisini R. (ed.) 2003, I piani della città. Trasformazione urbana, identità politiche e sociali tra fascismo, guerra e ricostruzione in Emilia-Romagna, Bologna: Compositori

Gobbo F. e Varni A. 1987, Dalla terra alla macchina: uno sviluppo nella tradizione, in Gobbo F. (ed.) 1987, Bologna 1937-1987: cinquant'anni di vita economica, Bologna: Cassa di Risparmio di Bologna

Grappi E. e Mammana S. 1988, Industria meccanica e modello emiliano. Innovazione, prodotti, organizzazione, Milano: Franco Angeli

Menzani T. 2011, La macchina nel tempo. La meccanica strumentale italiana dalle origini all'affermazione in campo internazionale, Bologna: Clueb

Menzani T. 2012, Progetti e ingranaggi. Il packaging e la meccanica strumentale nella storia di Bologna e dell'Emilia-Romagna, in De Maria C. (ed.) 2012, Bologna Futuro. Il "modello emiliano" alla sfida del XXI secolo, Bologna: Clueb

Menzani T. 2015, Tradizione, innovazione, automazione. Il packaging bolognese nel terzo millennio: una breve disamina quantitativa, "Scuola Officina", 1-2

Menzani T. 2013, Bologna la ricca. Uno sguardo al tessuto socio-economico cittadino negli anni in cui nacque la Fiera del Libro per Ragazzi, in Grilli G. (ed.) 2013, Bologna. Cinquant'anni di libri per ragazzi da tutto il mondo, Bologna: Bononia University Press

Micheli G.A. 1999, Effetto generazione: cinquant'anni di trasformazioni demografiche in Italia dal dopoguerra a oggi, Roma: Carocci 
Parisini R. 2010, Consumi e trasformazioni urbane a Bologna (1951-1981). Prime considerazioni di una ricerca, in Varni A. e Parisini A. (eds.) 2010, Consumi e trasformazioni urbane tra anni Sessanta e Ottanta, Bologna: Bononia University Press

Pedrocco G. 2013, Bologna industriale, in Varni A. (ed.) 2013, Storia di Bologna, vol. 4, Bologna in età contemporanea, tomo II, 1915-2013, Bologna: Bononia University Press

Petroni G. 1997, Cultura tecnologica e sviluppo dell'impresa. Cinque casi di grandi innovazioni industriali italiane, Padova: Cedam

Polanyi K. 1974, La grande trasformazione, Torino: Einaudi (ed. or. 1944)

Romei V. 2006, Terziarizzazione manifatturiera e integrazione dei servizi in Italia (18911985), "Imprese e storia", 33

Serafini M. 2014, Machanics "Made in Italy”: Innovation and Expertise Evolution. A Case Study from the Packaging Industry, 1960-1998, "History of Technology", 32

Spagnolo C. 2001, La stabilizzazione incompiuta. Il piano Marshall in Italia, 1947-1952, Roma: Carocci

Zamagni V. 1980, L'economia, in Zangheri R. (ed.) 1980, Bologna, Roma-Bari: Laterza

Zamagni V. 1997, Una vocazione industriale diffusa, in Finzi R. (ed.) 1997, L'Emilia Romagna, Torino: Einaudi 